\title{
Geometrical shock dynamics and engine unstart
}

\author{
Prasanna A.Varadarajan* and Philip L.Roe ${ }^{\dagger}$ \\ Department of Aerospace Engineering, University of Michigan, Ann Arbor, MI , USA 48109
}

In this work we contribute to the study of the engine "unstart" problem on the assumption that noise caused by shock-induced separation of the turbulent boundary layer can contribute to the problem. This noise is caused by strong discrete vortices encountering the foot of the reflected shock. It can be transmitted along the shock. We have made extensions to the theory of Geometrical Shock Dynamics that permit very rapid estimates of this noise up to distances of 400 times the core radius of the vortex, and find that in some cases there is only slight reduction of amplitude. These calculations are validated by comparing them, for shorter distances, with solutions obtained from the Euler equations.

\section{Introduction}

The work to be described here had its origins in the practical problem of "engine unstart" This term relates to the fact that airbreathing engines fitted to a supersonic vehicle need to have the inlet flow compressed to a subsonic state before it enters the engine. This is commonly achieved by sending the air through a series of oblique shockwaves, which reflect between the walls of the intake. For hypersonic flight, the number of shocks may be quite large, and the setup is described as a "shock train". Such a train is illustrated in Figure 1.

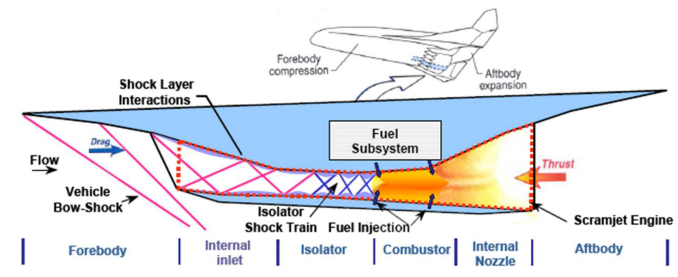

Figure 1. Representative Scramjet engine

These shock trains are prone to break down and collapse, and the reason for this remains mysterious. Our work began in an attempt to investigate if the shocks could be subjected

${ }^{*}$ Graduate Research Assistant

$\dagger$ Professor, Fellow AIAA 
to strong oscillations originating in the interaction of a reflected shock with a turbulent boundary layer. It has been known for some time that such a reflection is characterized by noise at a frequency considerably below the frequencies found in the approaching boundary layer. A mechanism for generating this noise was proposed by Pirazzolli and Grasso ${ }^{9}$ on the basis of very detailed DNS simulations. These showed that the separation bubble found beneath the reflected shock was highly unsteady. Its boundary is marked by a series of strong discrete vortices that meet with the foot of the shock, and cause a large displacement and distortion of the shockwave, as shown in Figure 2. The rms variation of Mach number in
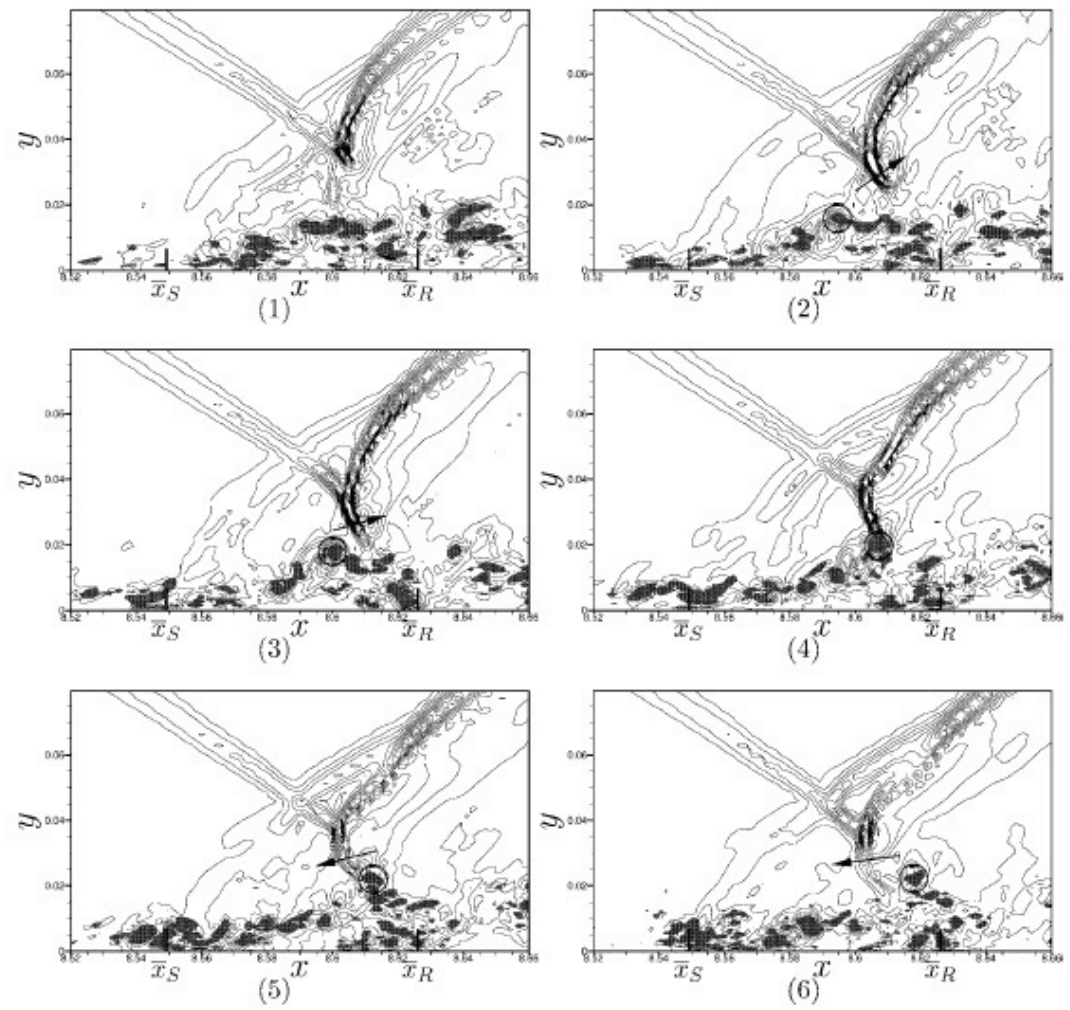

Figure 2. DNS results of Pirozolli and Grasso ${ }^{9}$ showing vortices interacting with the pulsating shock at different time intervals. Plots of the gradient of the pressure field at different times

this region is about 0.35 , so some perturbations are much greater than this. The simulations also showed waves propagating along the shock surface in a very two-dimensional manner as in Fig 3.

The waves appear to diminish in amplitude and increase in wavelength as they leave the region. It was initially unclear whether these waves were physical or numerical in origin. Assuming that they are physical it is natural to ask how far they may travel. For example, if they are capable of reaching the site of the next reflection, they might act as a forcing effect at the resonant frequency. It would be difficult to investigate this numerically because the waves would have to propagate over many times their wavelength (which seems to be of the order of the boundary layer thickness) Therefore we looked into the possibility of investigating the problem by simpler methods.

Specifically we looked at Whitham's theory of Geometrical Shock Dynamics, ${ }^{18}$ proposed in 1957 as a way to investigate the propagation of a shock without needing to know all of (indeed, any of) the postshock flow. We hoped to find solutions that could be verified 


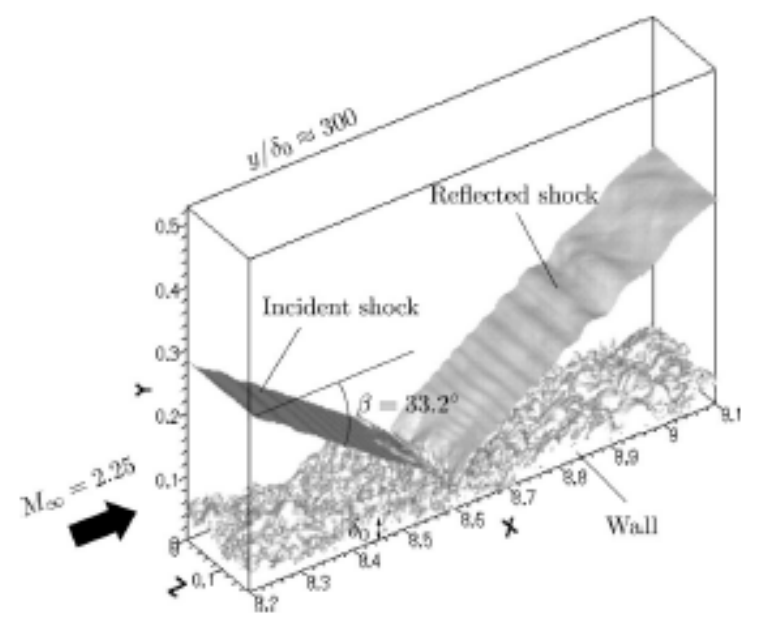

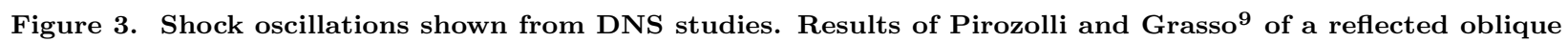
shock configuration

by Euler calculations, and hoped to establish the asymptotic rate of decay of these waves. We were actually successful in this, but only after making substantial enhancements to the Whitham theory. The great advantage of this theory, in its original form, was that it reduced the dimensionality of the problem. Disturbances to two-dimensional shocks are governed by one-dimensional equations, and perturbations to three-dimensional shocks are governed by two-dimensional equations. Additionally the equations have fewer variables, so that a reduction of orders of magnitude in computing time can be anticipated and was infact achieved.

However, the theory in this form had only been formulated for two-dimensional shocks propagating into a stationary fluid. It had not been found possible to retain these advantages if the shock propagated into a moving fluid, or was three-dimensional. In these cases no reduction in dimensionality had been achieved. Rather surprisingly, we found very simple methods, apparently overlooked, that restored the simplicity of the original formulation to these new contexts.

We than applied the two-dimensional theory to the problem of shock vortex interaction. We realized that the Whitham theory had little hope of modeling the initial stages of the interaction, but hoped that it would predict how the waves would behave at greater distances. We were able to show the existence of waves that decay rather slowly (like the inverse square root of distance). Calculations with the full Euler equations have confirmed that such waves are indeed created under some circumstances.

We have an incomplete version of the three-dimensional code, but not yet any significant results from it. However, a consideration of qualitative features of the three dimensional problem already indicates the possibility of very strong coupling between different parts of the shock train.

\section{Whitham's Geometrical Shock Dynamics}

Whitham ${ }^{18}$ proposed GSD in 1957 as a way to study the evolution of shockwaves without needing to know any details of the flow behind them. At that time, he considered only the 


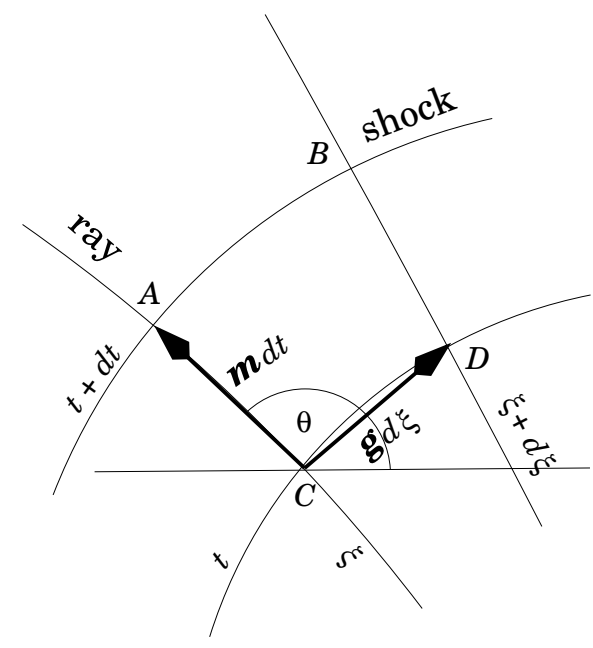

Figure 4. Part of the net of shock locations and rays.

case of a shock advancing into stationary fluid and we will, to begin with, do the same. He introduced coordinates $(\xi, t)$ such that curves $t=$ const represent snapshots of the shockwave at the corresponding time, and curves $\xi=$ const are orthogonal to the snapshots. Each curve $\xi=$ const is called a ray and the region between two closely spaced rays is called a raytube. An economical derivation of the basic result comes from defining vectors $\mathbf{m}(\xi, t)$ and $\mathbf{g}(\xi, t)$ such that elements of the coordinate system are given by $\mathbf{g}(\xi, t) d \xi$ and $\mathbf{m}(\xi, t) d t$. The magnitude $m$ of $\mathbf{m}$ is the speed with which the shockwave advances normal to itself, and with a suitable choice of units is the normal Mach number. The vector representing an arbitrary line element is $d \mathbf{r}=\mathbf{g} d \xi+\mathbf{m} d t$ so that around any closed curve we have

$$
\oint \mathbf{g} d \xi+\mathbf{m} d t=0
$$

This has the appearance of a conservation law, ${ }^{1}$ with $\mathbf{g}$ as the conserved quantity that advances in time due to the gradient of a flux function $\mathbf{m}$. Discretisation of the conservation law is trivial; around the cell $A B C D$ we have

$$
\left(\mathbf{r}_{A}-\mathbf{r}_{B}\right)-\left(\mathbf{r}_{C}-\mathbf{r}_{D}\right)=\left(\mathbf{r}_{A}-\mathbf{r}_{C}\right)-\left(\mathbf{r}_{B}-\mathbf{r}_{D}\right)
$$

To make this useful, physics must be added in the form of some relationship $m(g)$. For example, if we simply take $m=$ const, we recover Huyghen's Principle. Whitham ${ }^{18}$ derived a flux function by assuming that the shock would propagate along each raytube as though the raytube were a narrow channel. This leads to a relationship of the form

$$
g / g *=f(m) / f(m *)
$$

where $m *, g *$ are (possibly fictitious) reference states.

Some justification for this was provided by Maslov ${ }^{11}$ and by Best, ${ }^{2,3}$ who showed that shockwave propagation could be rigorously represented by a hierarchy of models, each of them correcting the previous model by adding information relating to one more normal derivative of the post-shock flow. From this viewpoint, the Whitham model could be regarded as the zeroth-order description. Prasad ${ }^{13}$ has claimed that at least one more derivative needs to 
be taken into account, and introduced a quantity $N$, being a lumped version of the normal derivatives. It is debatable how useful this is, because in most cases it is difficult to supply data for this variable. Therefore, we follow Whitham by seeking a simple closure $m(g)$. If one is available, then we can rewrite 1 as a nonconservative pair of hyperbolic equations

$$
\begin{aligned}
\partial_{t} \theta+\frac{m^{\prime}(g)}{g} \partial_{\xi} g & =0 \\
\partial_{t} g-m(g) \partial_{\xi} \theta & =0
\end{aligned}
$$

whose characteristic speeds are $\lambda_{1,2}= \pm \sqrt{\frac{-m(g) m^{\prime}(g)}{g}}$ in the $\xi-t$ coordinates. In the physical coordinates the wavespeeds are $c_{1,2}= \pm \sqrt{-m(g) m^{\prime}(g) g}$.

A weakness of Whitham's closure is that it badly underpredicts (by a factor of two) the speed with which waves travel along a weak shock. We propose a new closure by matching these wavespeeds to the actual speed with which a disturbance propagates along a shockwave. Simple geometry gives the exact speed of propagation $c$ along the shock as

$$
c^{2}=a_{2}^{2}-u_{2}^{2}
$$

where we have from the exact shock relationships that

$$
\frac{u_{2}}{u_{1}}=1+\frac{2\left(1-M_{1}^{2}\right)}{(\gamma+1) M_{1}^{2}}, \quad \frac{a_{2}^{2}}{a_{1}^{2}}=\frac{\left(2+(\gamma-1) M_{1}^{2}\right)\left(\frac{2 \gamma}{\gamma-1} M_{1}^{2}-\frac{\gamma-1}{M_{1}^{2}}\right)}{(\gamma+1)^{2}}
$$

Then setting

$$
g^{2} \lambda^{2}=c^{2}
$$

gives a differential equation

$$
\frac{g^{\prime}}{g}=-\frac{(\gamma+1) m^{3}}{\left((\gamma-1) m^{2}+2\right)\left(m^{2}-1\right)}
$$

that can be integrated to give

$$
g(m)=\frac{\left(2+m^{2}(\gamma-1)\right)^{\frac{1}{1-\gamma}}}{\sqrt{m^{2}-1}}
$$

The propagation speeds according to this model and the original Whitham model are shown in Figure II, together with a relation derived by Prasad for weak shocks

$$
g(m)=(m-1)^{-2} e^{(-2(m-1))}
$$

It can be seen that the present, exact, result agrees with Prasad's model at low Mach numbers, and with Whitham's model at high Mach numbers.

In fact, experiments show that any of these closures produces a model with very similar qualitative behavior, and since no version of GSD can be used for more than qualitative prediction, the choice of closure does not matter greatly. Results here use the new closure, because it is considerably simpler than Whitham's, and at high Mach numbers more accurate than Prasad's. 


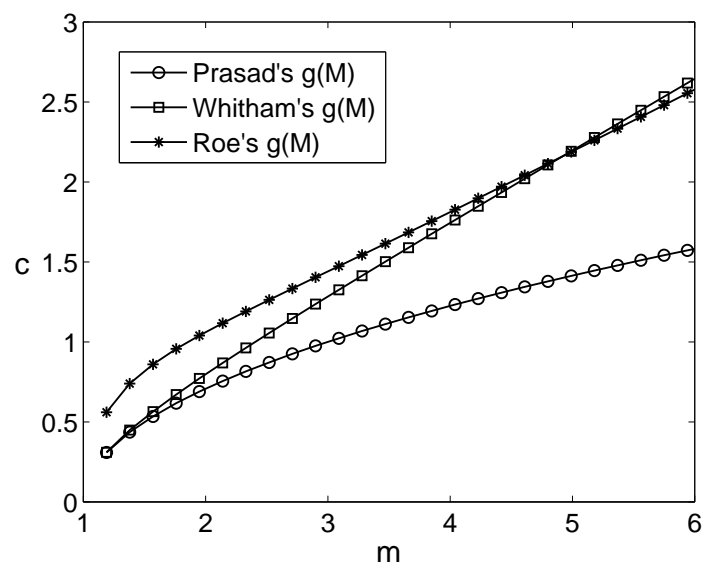

Figure 5. Wave-speeds in the physical space along the shock

\section{II.A. Numerical treatment of GSD}

Our numerical solution of GSD follows the usual lines of "high-resolution" shock-capturing finite-volume methods, ${ }^{8}$ but with a distinctly geometrical flavor. We use the discrete form of the geometrical conservation law (equation 2) and the numerical task is to estimate a typical "flux vector" such as QT. The magnitude and direction of this vector are simply $m$ and $\theta$, which are found by solving a Riemann problem with $m, g(m)$ and $\theta$ in the neighboring cells as data, as shown in Figure 6.

A detail that is worth pointing out is that the derivation of an approximate Riemann solver is simplified when the wavespeeds in the problem are equal and opposite $( \pm \lambda)$, as they are in GSD. In this case, the viscosity matrix can be simplified thus;

$$
\mathbf{Q}=|\mathbf{A}|=\mathbf{R}|\mathbf{\Lambda}| \mathbf{L}=\mathbf{R}(|\lambda| \mathbf{I}) \mathbf{L}=|\lambda| \mathbf{I}
$$

and the Roe dissipation reduces to a special case of the much simpler Rusanov dissipation.

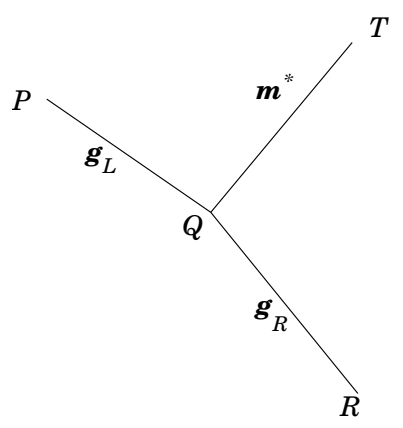

Figure 6. A geometrical Riemann problem. To advance the ray QT, we need information from the neighboring "cells" PQ,QR, which is used to formulate a Riemann problem with $m, g(m), \theta$, as data.

\section{II.B. Propagation into a moving stream}

This ought to be a simple extension of this method, to shocks propagating into a stationary fluid, but it has proved troublesome in the past. Whitham, ${ }^{20}$ noting that an attempt by Chisnell ${ }^{4}$ did not satisfy Galilean invariance, abandoned much of the geometrical character 
of GSD. He introduced a function $\alpha(x, y)$ whose level lines are the snapshots of the shockwave at $t=\alpha$, and derived a second-order partial differential equation satisfied by $\alpha$. He noted that this could be solved iteratively by methods similar to those used for transonic potential flow.

This change of viewpoint has three unfortunate consequences. Firstly, a good deal of the intuitive clarity of GSD is lost. Secondly, the numerical method required is two-dimensional rather than one-dimensional. The reduction in dimensionality has been lost, and indeed, it is probably about as hard to write a good code for the $\alpha$-equation, as to write one for the unsteady flow equations themselves (and these days, the latter codes are available off the shelf). Thirdly, and this is particularly relevant to our application, it becomes very hard to solve flows that are almost steady, because in such cases the shockwave is likely to pass over the same location $(x, y)$ more than once. The function $\alpha(x, y)$ would be multivalued at such points, greatly complicating it's computation.

Fortunately, there is actually a very simple solution that seems to have been overlooked. We note that propagation into a moving stream, seen by a stationary observer, is the same as propagation into a stationary fluid viewed by a moving observer. We can think of the intersection of a particular ray with a particular snapshot of the shockwave as an event that might be viewed by either observer. All that has to be done is to compute the solution $\mathbf{m}(\xi, t)$ as though the fluid were at rest, and then to advance the solution from $t$ to $t+d t$ in the physical plane by means of

$$
\frac{d \mathbf{x}}{d t}=\mathbf{m}+\mathbf{m}_{0}
$$

where $\mathbf{m}_{0}$ is the velocity of the moving stream.

\section{Modeling shock-vortex interaction}

\section{III.A. The GSD model}

We consider an inviscid vortex convected by a uniform stream toward a normal shock. ${ }^{\text {a }}$ When the vortex arrives at the shock the velocities on one side of the vortex will augment the free stream, so that the shockwave will in effect be moving faster into a uniform stream, and this will be reversed on the opposite side. We propose to model the encounter by prescribing an initial-value problem for $m$ as a function of $\xi$. Specifically we prescribe

$$
\begin{aligned}
m(\xi, 0) & =m_{o}\left(1-C \sin \frac{\pi(L \xi)}{2 r_{v}}\right), \quad|\xi|<\frac{2 r_{v}}{L} \\
& =m_{o}, \quad|\xi|>=\frac{2 r_{v}}{L}
\end{aligned}
$$

where $r_{v}$ imitates the vortex core radius and L a specific length of the shock considered. This gave quite realistic results without any accompanying perturbation in $\theta$, and since we can only hope for qualitative predictions it did not seem worthwhile to refine it.

This initial data applies equal but opposite perturbation to the two Riemann invariants, and this will result in two simple waves propagating to left and right along the shock. If

\footnotetext{
aThe case of an oblique shockwave is handled by superposing a velocity parallel to the shock
} 
the propagation were governed by linear equations, they would remain equal but opposite. However, nonlinear aspects of their propagation will be different. On the side where the vortex augments the free stream, represented here by an increase in $m$ on the left, we have a compression followed by an expansion followed by a compression. This is the usual N-wave, but we call it a C-E-C wave to distinguish it from the E-C-E wave on the other side, where an initial expansion is followed by a compression and then a second expansion. We observed that the E-C-E and C-E-C waves often displayed quite different asymptotic behavior. A sample calculation is shown in Figure 7 . Note that the $\mathrm{C}-\mathrm{E}-\mathrm{C}$ wave grows in length but the E-C-E wave does not. Note also that the E-C-E waves decay much faster.
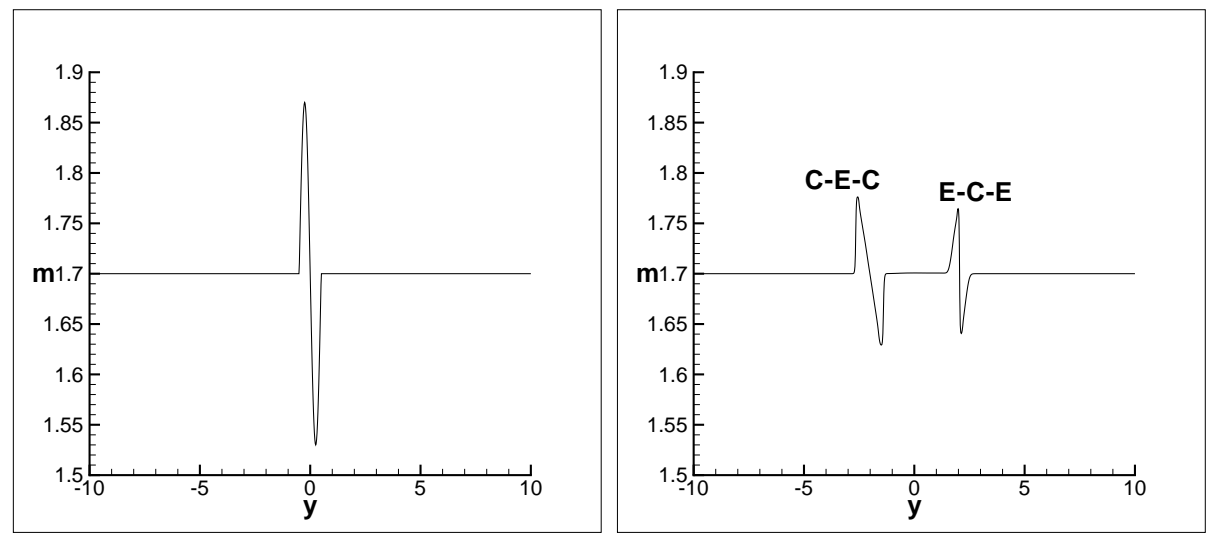

(a) Initial $m$ distribution along the normal shock front

(b) $m$ distribution along the shock after

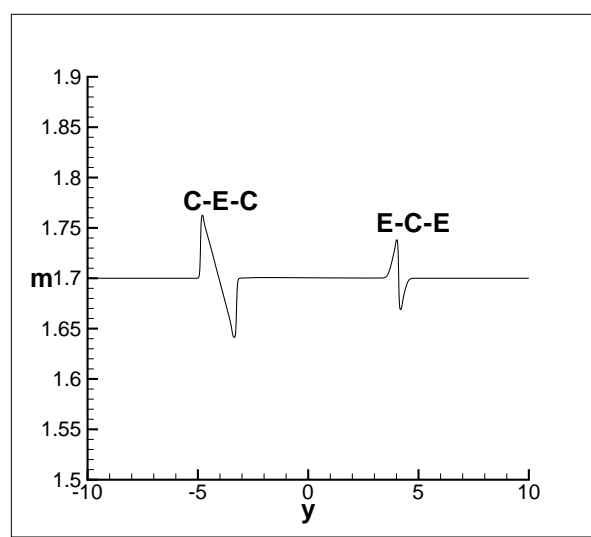
$\mathrm{t}=2.2$ units

(c) $m$ distribution along the shock after (d) m distribution along the shock after $\mathrm{t}=4.4$ units $\mathrm{t}=6.6$ units

Figure 7. GSD results : At early times using our closure model for a planar shock of strength 1.7

\section{III.B. The two wave types}

To explain this, we examined the difference in behavior for each type of wave in Burgers' equation. This is justified by the fact that small amplitude waves in any set of equations satisfy Burgers' equation in smooth regions, and weak shocks are not very different. ${ }^{16}$ Burgers'equation is of course

$$
\partial_{t} u+u \partial_{x} u=0
$$


By employing the properties that $u=$ const along characteristics $d x / d t=u$, and that shocks move at a speed $d x_{s} / d t=\left(u_{L}+u_{R}\right) / 2$ exact solutions can be found for the two problems whose initial data is displayed in Figure 8. We omit the straightforward details, and simply present results.

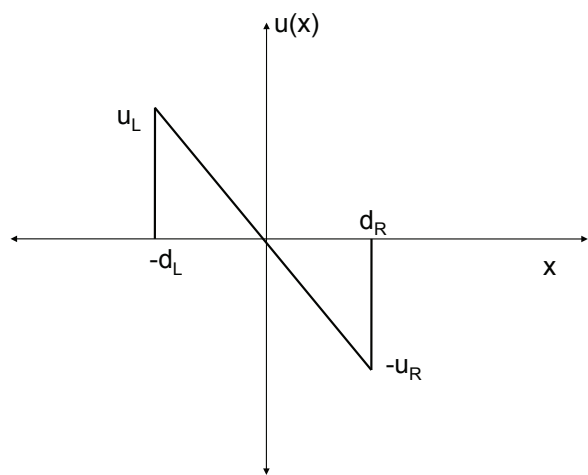

(a) C-E-C wave

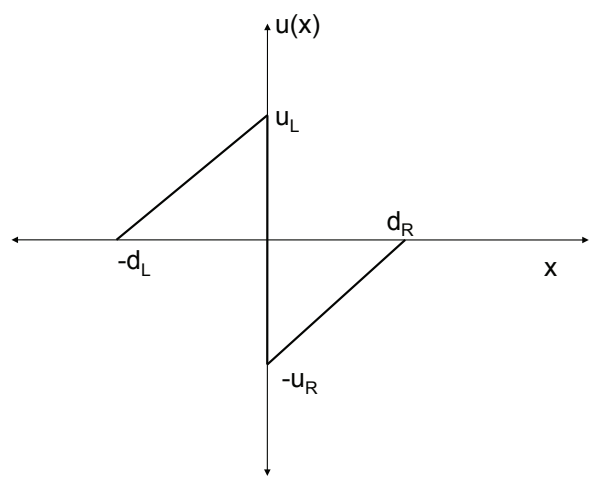

(b) E-C-E wave

Figure 8. Initial data for the generic wave structure

The C-E-C WAve This is the well-known N-wave; both shocks recede outward, and the solution remains linear between them. Formulas for the shock strengths as a function of time are;

$$
u_{L, R}(t)=\left(u_{L, R}(0)\right)\left[\left(1+\frac{u_{L, R}(0) t}{d_{L, R}}\right)\right]^{-1 / 2}
$$

In particular we see that both shocks decay at an asymptotic rate of $t^{-1 / 2}$.

The E-C-E WAVE This is more complicated, and less well-known. Initially, the solution is confined within the range $-d_{L}<x<d_{R}$, and the shock moves to the right or to the left depending on the sign of $u_{L}(0)-u_{R}(0)$. However the shock may not always continue moving in the same direction. There are three cases depending on the value of

$$
I_{0}=\int_{-\infty}^{\infty} u(x, 0) d x=u_{L}(0) d_{L}-u_{R}(0) d_{R}
$$

If $I_{0}$, which is integral of the initial data, is negative then the shock, in finite time, leaves the initial range through the left boundary; if $I_{0}=0$ it remains for all time within the initial range, and for $I_{0}>0$ it leaves on the right. Before the shock leaves, its strength is given by the monotone decreasing function

$$
u_{L}(t)-u_{R}(t)=\left(u_{L}(0)-u_{R}(0)\right)\left[\left(1+\frac{u_{L}(0) t}{d_{L}}\right)\left(1+\frac{u_{R}(0) t}{d_{R}}\right)\right]^{-1 / 2}
$$

which decreases for large $t$ like $t^{-1}$. If the shock is going to leave, it does so at a time given by

$$
t^{*}=\frac{1}{\left|I_{0}\right|}\left[\left\{u_{L}(0) d_{R}+u_{R}(0) d_{L}\right\} \max \left(\frac{d_{R}}{u_{L}(0)}, \frac{d_{L}}{u_{R}(0)}\right)+2 d_{L} d_{R}\right]
$$


At this time, it can be shown that either $u_{L}\left(t^{*}\right)=0$ or $u_{R}\left(t^{*}\right)=0$. We then have a new "initial-value" problem, consisting of a single shock and a single rarefaction. The shock now leaves the initial range, departing from it by a distance proportional to $\left(t-t^{*}\right)^{1 / 2}$ and weakening as $\left(t-t^{*}\right)^{-1 / 2}$.

In summary, the extent of the wave does not initially increase, and the weaker of the two initial disturbances vanishes after finite time. The stronger of the two decays initially like $t^{-1}$, but after the weaker disturbance disappears, like $t^{-1 / 2}$. In this final stage, the waves extend beyond their original support. These distinct behaviors for the two types of wave correspond quite closely to what we observed in the GSD solutions, and also in at least some of the Euler simulations.

\section{A note on oblique shocks}

The discussion so far has been in terms of a normal shock, and the results will be presented in this context also. However the interaction of a single vortex with an oblique shockwave is merely a uniform translation of the normal shock case, and therefore introduces no new theory. A practical matter is that if the flow is supersonic behind an oblique shock, then both types of wave are propagated in the same direction as a fast wave and a slow wave. The decay with respect to time is unchanged, but the fast waves will travel for a greater distance. For the situation depicted in the figure 2, clockwise vortices interact with the shock, each encounter producing a fast going $\mathrm{E}-\mathrm{C}-\mathrm{E}$ wave and a slow going $\mathrm{C}-\mathrm{E}-\mathrm{C}$ wave. The distinction between waves that travel in both directions, or only in one, is made correctly only if the wavespeed along the shock is correct. That is another motivation for choosing the new closure (6).

\section{Results from GSD}

Realizing that the C-E-C waves continually grow in extent and diminish in amplitude explains the behavior of the waves seen in Figure 3. We now turn to the issue of decay at larger distances, and present a some long range results obtained from the GSD model, tracking the disturbances out to about $400 r_{v}$. We tested a range of shock strengths (the unperturbed initial value of $m$ ), and various values of $C$ in equation (9), but give here only a small representative sample. We were particularly interested to determine the rate at which signals decayed as they traveled away from the site of the encounter. If the decay is slow, then this phenomenon is more likely to be of practical importance. We made log-log plots showing, as function of distance from the origin, the maximum and minimum (i.e. most negative) strengths of the waves. That is, at a given location, what is the greatest perturbation experienced? The results have been presented in terms of the pressure perturbation. There was a notable difference between stronger and weaker initial perturbations. In Figure 9d, we see all of the expected behaviors. Both amplitudes of the $\mathrm{C}-\mathrm{E}-\mathrm{C}$ wave decay consistently like $t^{-1 / 2}$. One branch of the E-C-E wave vanishes and the other eventually decays like $t^{-1 / 2}$ after decaying more quickly at earlier times. As we expect, the weaker the perturbation, the later the waves take on their asymptotic behavior. The weakest waves did not decay at all within the computational domain; the smooth initial profiles had not yet formed shockwaves.

10 of 16

American Institute of Aeronautics and Astronautics 


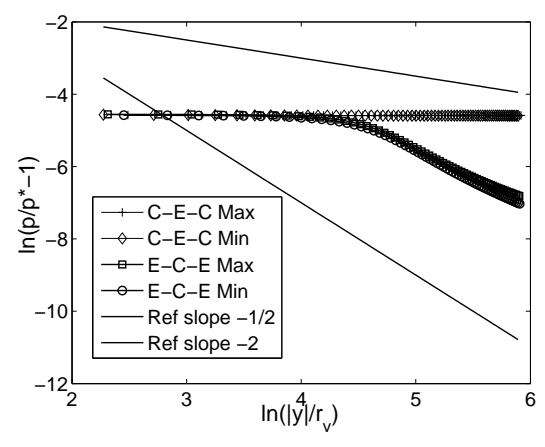

(a) $\delta \mathrm{m}=1 \%$ of $\mathrm{m}$

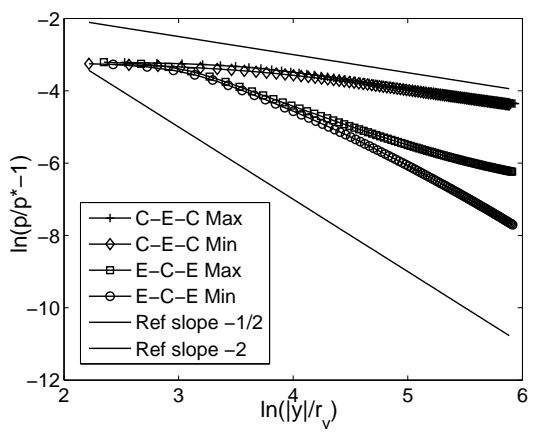

(c) $\delta \mathrm{m}=4 \%$ of $\mathrm{m}$

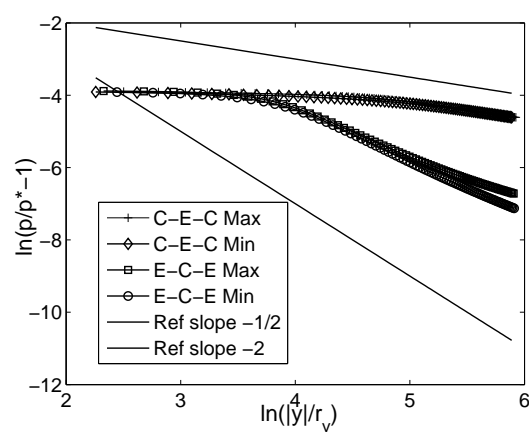

(b) $\delta \mathrm{m}=2 \%$ of $\mathrm{m}$

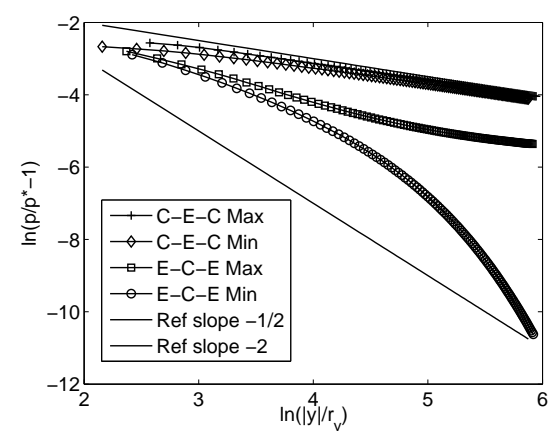

(d) $\delta \mathrm{m}=8 \%$ of $\mathrm{m}$

Figure 9. GSD results : Power law decay of the wave amplitudes with non dimensional distance for shock with $\mathrm{m}=1.7$. $\mathrm{p}^{*}$ represents the downstream pressure

\section{Results from Euler simulations}

Similar experiments were conducted with the Euler equations, using a standard secondorder high-resolution method. A square mesh having 250 by 750 cells was used, with the vortex core(the edge of which the vortex has the maximum velocity) initially having a diameter of 25 cells. Simple outflow boundary conditions proved satisfactory. This allowed the vortex to be tracked over a distance of about $25 r_{v}$, not far enough to reach a convincing asymptotic regime, but far enough to confirm some of our expectations based on GSD. The calculations were not quite grid-converged, but we felt confident that refining the grid would not greatly affect our conclusions, although it would have curtailed the number of experiments that we could do. A typical run took about half an hour, compared with less than a second for a GSD run, confirming 3 orders of magnitude less computation. A plane stationary shock was placed halfway across the grid, with a vortex ahead of it having a prescribed distribution of circumferential velocity till $4 r_{v}$ units. The nature of this distribution only had a small effect on our results, although it is reported to be significant in the near field. ${ }^{5}$

To begin with, we visualized the developing flow by drawing contour lines of the flow quantities. These were not very informative. Particularly it proved very difficult to see any distortion of the shock shape except close to the interaction. This may account for the fact that some authors have stated that the waves decay very rapidly. Certainly the perturbations do decay quickly in the near-field, but there may only be slow decay in the far field. We experimented with various ways to reveal this, eventually deciding to monitor events in a single line of cells located five cells behind the initial position of the shock (along AB shown 


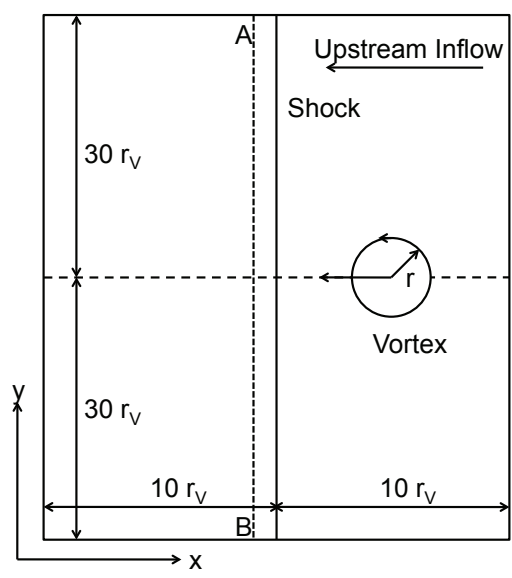

Figure 10. Computational domain for the shock vortex interaction with $r$ the outer radius of the vortex and $r_{v}$ the radius of the vortex core

in figure 10). Five was not a magic number, and other choices gave similar results, but much more than five resulted in weaker signals, and fewer resulted in noisier ones.

Again we investigated various shock Mach numbers $\left(M_{s}=1.05,1.2,1.4,1.7\right.$, and 2.0) and varying vortex strengths between 0.1 and 1.0 (defined as the ratio of the maximum circumferential velocity to the speed of sound in the free stream, and denoted by $M_{v}$ ) To place these numbers in perspective, we may consider the DNS simulations of shock/boundary-layer interaction conducted by Pirozolli and Grasso ${ }^{9}$ as shown in figures 2 and $3^{\mathrm{b}}$. We are grateful for a private communication to the effect that r.m.s fluctuations of Mach number were about 0.35 in the region traversed by the vortex. Since the maximum fluctuation will be greater than the r.m.s. fluctuation, we think that our range of vortex Mach numbers is not unrealistic.

The near field of the shock-vortex interaction has been studied numerically in ${ }^{5-7,10,14,21}$ and elsewhere. These authors have observed a classification based on the topology of the shock deformations. The shock either remained smooth, or formed two intersecting branches, or the branches connected through a Mach stem. The three different types were found in three distinct regions of the $\left(M_{s}, M_{v}\right)$ plane. We verified this classification as one way to validate our code, but found that this classification had little to say about the far-field behavior.

A representative sample of our results is presented in Figure 11 showing the pressure along the line $\mathrm{AB}$ at four different times. The results are definitely similar to those from GSD. Even though they are not nearly as "clean", we see a distinct asymmetry between the left- and right-going waves, with the right-going E-C-E waves decaying faster, and the left-going C-E-C waves growing in extent. In Figure 12, we present plots showing amplitude decay in the same way that we did for the GSD results ${ }^{c}$. A much larger set of results in available in the first authors thesis, ${ }^{17}$ but these three represent the three broad types of result that we found. We begin with the combination of a strong shock and a weak vortex (here $\left.M_{s}=2.0, M_{v}=0.1\right)$. These were the cases that most closely followed the prediction of the Whitham theory, and where there is strong asymmetry between the two types of wave. We

\footnotetext{
${ }^{\mathrm{b}}$ It was this paper that stimulated our interest in the problem.

${ }^{\mathrm{c}}$ Although here the pressure amplitude has been measured relative to the pressure difference found in the initial vortex.
} 
can see that one branch of the $\mathrm{C}-\mathrm{E}-\mathrm{C}$ wave is decaying like $t^{-1 / 2}$, while the other branch has not yet begun its asymptotic decay. Both branches of the E-C-E wave are decaying rapidly. The opposite case is that of a weak shock and a strong vortex (here $M_{s}=1.4, M_{v}=0.8$ ) where, although there is some asymmetry, all waves are decaying roughly like $t^{-1}$. Results for an intermediate case $\left(M_{s}=1.7, M_{v}=0.4\right)$ are appropriately intermediate in behavior.
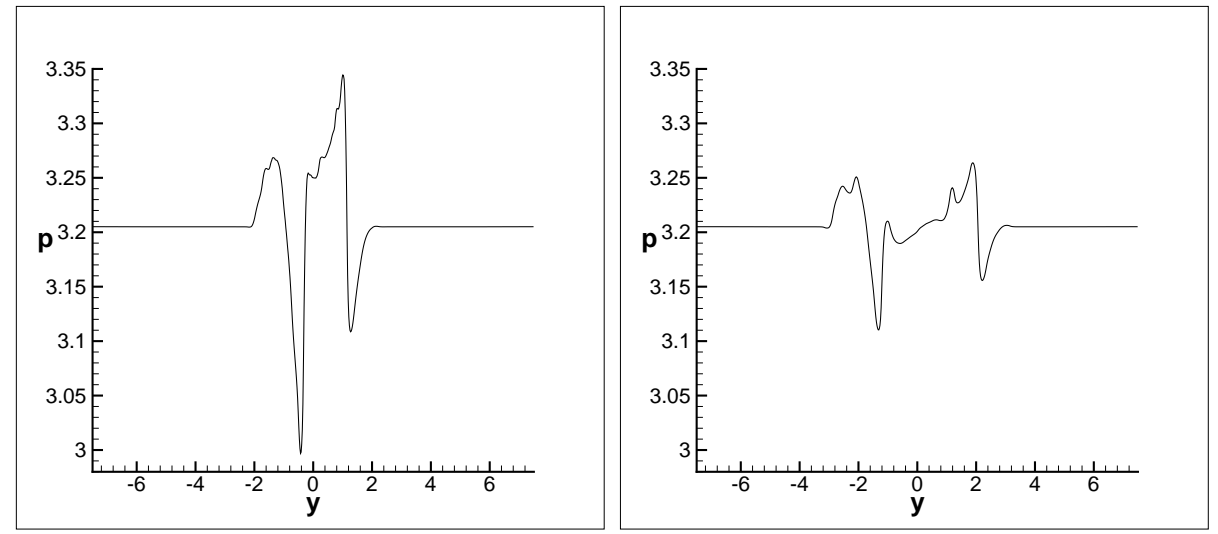

(a) pressure signal at time $\mathrm{t}=2$ units. (b) pressure signal at later time $\mathrm{t}=3$ The vortex passes through the shock units approximately at $\mathrm{t}=1.5$ units.
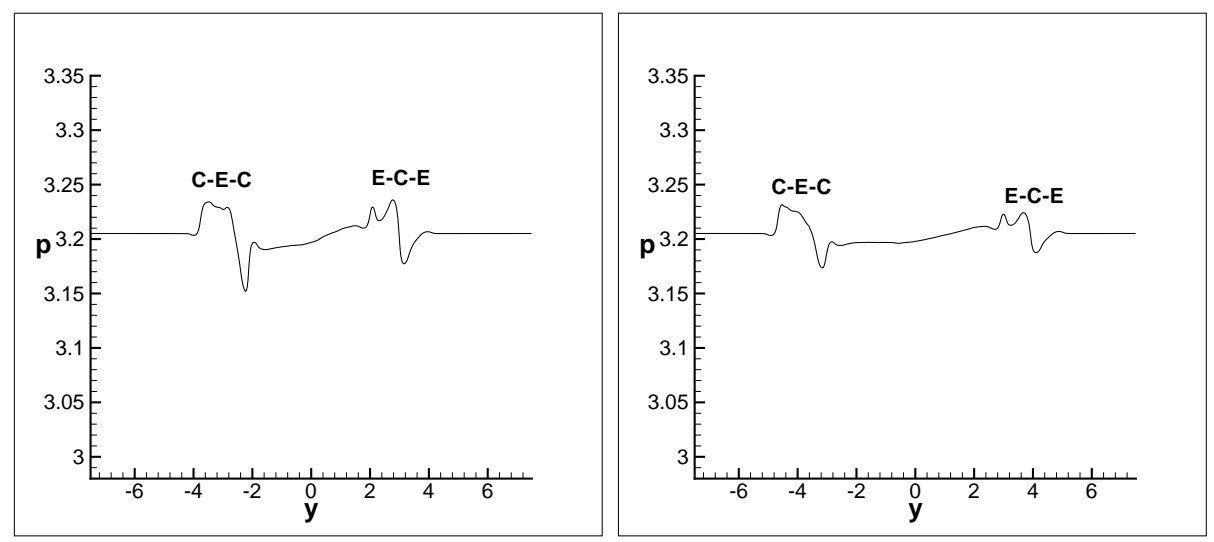

(c) pressure signal at a later time at $\mathrm{t}(\mathrm{d})$ pressure signal at still further time $=4$ units at $\mathrm{t}=5$ units

Figure 11. Euler results : Monitored pressure along $\mathbf{A B}$ for $M_{s}=1.7$ and $M_{v}=0.4$

As a check on our results, this last case was kindly run by Prof. Farhad Jaberi of Michigan State University, using a fifth-order method on a mesh of 1500 by 1500 cells. He obtained similar results for the behavior of the C-E-C wave, but a less rapid decay for the E-C-E wave. This indicates that this particular result of ours may not have been fully resolved. However, we definitely stand by our general conclusions.

Much of the literature on shock-vortex interaction deals with the acoustic wave shed by the vortex. As the circular vortex passes through the shock wave, it is compressed in the flow direction, taking on a roughly elliptical shape. This rotating ellipse throws off noise that is initially dipolar, and subsequently quadrupolar. Rault et. al. ${ }^{14}$ conducted high-resolution computations verifying theoretical predictions by Ribner ${ }^{15}$ that the precursor wave decays like $t^{-1}$ and the later wave (second sound) like $t^{-1 / 2}$. Simple geometry shows that the waves that propagate along the shock coincide with the reflection of the precursor wave from the 


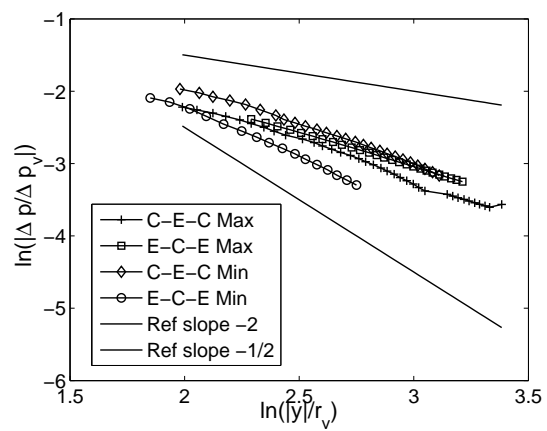

(a) $M_{s}=1.4$ and $M_{v}=0.8$

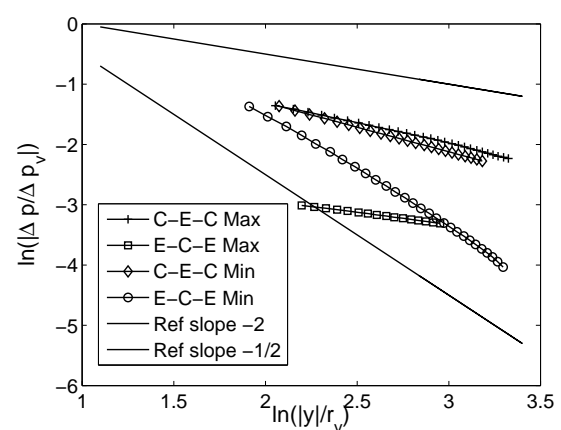

(b) $M_{s}=1.7$ and $M_{v}=0.4$

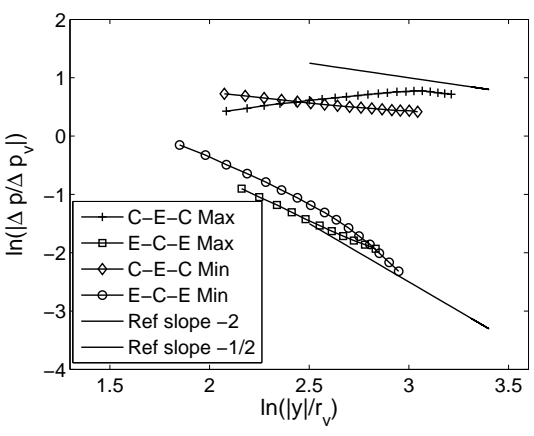

(c) $M_{s}=2.0$ and $M_{v}=0.1$

Figure 12. Power law decay of amplitudes of the pressure signal with $\Delta p_{v}$ being the strength of the pressure disturbance with the vortex before interaction

shock. Further work is needed to uncover the link between the two types of wave, but it is quite remarkable that so much of the actual physics can be obtained from the simple Whitham theory. This is enough to encourage the development of GSD in three dimensions.

\section{Three dimensions}

We have developed a three-dimensional version of GSD, free from the computational defects of the original, ${ }^{19}$ although we are not yet ready to present details of the computational scheme. Instead, we note some physical features. Figure 13 shows an oblique shockwave on whose surface an event $\mathrm{E}$ is taking place. News of this event is confined to the interior of the Mach cone centered on ES, but part of this cone is cut off by the shock surface. The part of the shock that is influenced by $\mathrm{E}$ is contained between the rays $\mathrm{ER}_{1}, \mathrm{ER}_{2}$. Just as the waves on a two-dimensional shock behave like one-dimensional waves, the waves on a three-dimensional shock surface behave like waves in two dimensions. If the surface of an initially plane oblique shock is described by coordinates $\xi, \eta$ then in steady flow there are characteristic lines

$$
\frac{d \eta}{d \xi}=\frac{1}{\beta}
$$

analogous to the characteristics of plane flow, for which $\beta^{2}=M^{2}-1$, but here, from the geometry of the figure $\beta^{2}=\left(u_{2}^{2}-a_{2}^{2}\right) / u_{n}^{2}$ Waves propagate within the shock surface just as waves propagate through a two-dimensional duct. If we now think of that shock as one element in a shock train, then it is in contact with a potentially unstable boundary layer on 

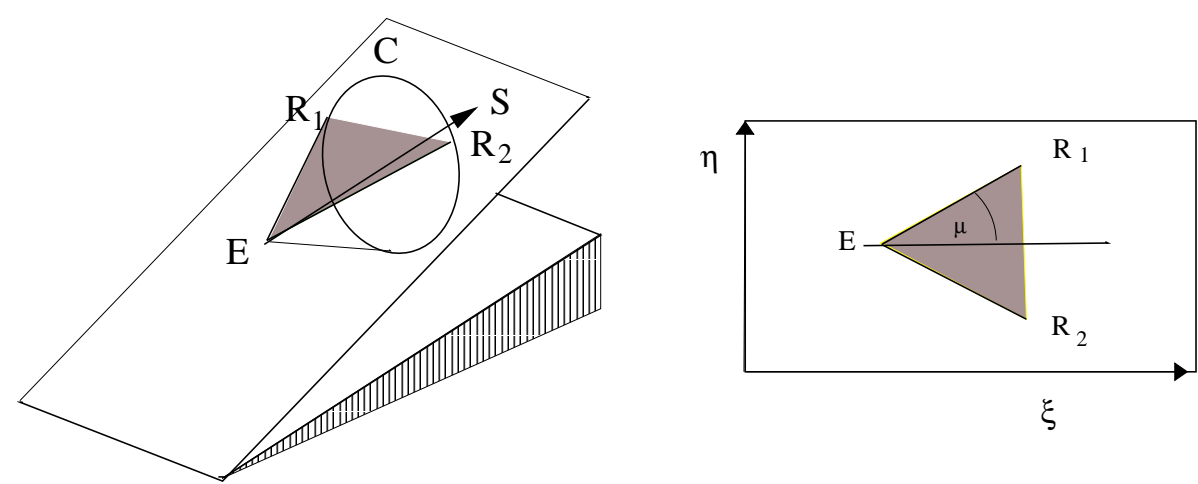

Figure 13. Characteristics along the shock surface

every edge. The shock surface can act as a waveguide for low-frequency noise at the resonant frequency of the separation bubbles.

\section{Conclusions}

We have rebuilt Whitham's theory of Geometrical Shock Dynamics on its original geometrical foundations, and applied it to the long range behavior of the interaction between a plane shockwave and a cylindrical vortex. The finding, confirmed for shorter ranges by Euler computations, is that noise can propagate along a shockwave following interaction with a vortex, and that under some circumstances it can do so for considerable distances. It is possible that this mechanism, or a three-dimensional version of it, plays a role in the "unstarting" of shock trains, by transmitting over a distance the violent fluctuations associated with shock-induced separation of the turbulent boundary layer.

\section{References}

${ }^{1} \mathrm{~S}$. Baskar,P. Prasad. Riemann problem for kinematical conservation laws and geometrical features of nonlinear wavefronts. IMA J. App. Math., 69, p. 391, 2004.

${ }^{2}$ J. P. Best. A generalisation of the theory of shock dynamics. Shock Waves, 1, p. 251, 1991.

${ }^{3}$ J. P. Best. Accounting for transverse flow in the theory of geometrical shock dynamics. Proc. Roy. Soc. Lond. Ser. A, 442, p. 585, 1993.

${ }^{4}$ R. F. Chisnell. A note on Whithams rule. J. Fluid Mech. 22 p.103, 1965.

${ }^{5}$ J. L. Ellzey, M. R. Henneke, The shock-vortex interaction: The origins of the acoustic wave, Fluid Dynamics Research, 21(3)p.171, 1997.

${ }^{6}$ J.L. Ellzey, M. R. Henneke, The acoustic wave from a shock-vortex interaction: comparison between theory and computation, Fluid Dynamics Research, 27(1), p 53, 2000.

${ }^{7}$ F. Grasso, S. Pirozzoli, Shock-WaveVortex Interactions: Shock and Vortex Deformations, and Sound Production, Theor. and Comp. Fl. Dynamics, 13, p. 421, 2003.

${ }^{8}$ R. J. Leveque, Numerical Solution of Conservation Laws, Birkhauserr, 1999.

${ }^{9}$ S. Pirozzoli, F. Grasso, Direct numerical simulation of impinging shock wave/turbulent boundary layer interaction at $\mathrm{M}=2.25$, Physics of Fluids,18, p. 065113, 2006.

${ }^{10}$ O. Inoue, Y. Hattori, Sound generation by shock-vortex interaction, j. Fluid Mech., 380, p.81, 1999.

${ }^{11}$ V.P. Maslov. Propagation of shockwaves in an isentropic non-vioscous gas. J. Sov. Math., 13, p.119, 1980.

${ }^{12}$ A. Powell, Sound Radiation from Spinning Vortices J. Acoust. Soc. Am., 35,(5), p.795, 1963. 
${ }^{13}$ P. Prasad. Nonlinear Hyperbolic Waves in Multi-Dimensions. Chapman and Hall/CRCJournal of Scientific Computing, Vol. 19, Nos. 13, December 2003 ( 2003)

${ }^{14}$ A. Rault, G. Chiavassa, R. Donat, Shock-vortex interactions at high Mach numbers, J. Sci. Comp., 19, p.347, 2003.

${ }^{15}$ H. S. Ribner, Cylindrical sound wave generated by shock-vortex interaction, AIAA Jnl., 23(11), p. $1708,1985$.

${ }^{16} \mathrm{~L}$. Ting, Transmission of singularities through a shock wave and the sound generation, Physics of Fluids, 17(8), p.1518, 1974.

${ }^{17}$ P.A. Varadarajan, Noise transmission along shockwaves, Ph. D. thesis, Aerospace Engineering, University of Michigan, June 2011.

${ }^{18} \mathrm{G}$. B. Whitham,A new approach to problems of shock dynamics Part I Two-dimensional problems, $J$ Fluid Mech., 2, p.145, 1957.

${ }^{19}$ G.B. Whitham, A new approach to problems of shock dynamics Part 2. Three-dimensional problems, J. Fluid Mech, 5, p369, 1959.

${ }^{20}$ G.B. Whitham. A note on shock dynamics in a moving medium. J. Fluid Mech., 31, p.449, 1968.

${ }^{21}$ S. Zhang, Y. T. Zhang, C-W Shu, Multistage interaction of a shockwave and a strong vortex, Phys. Fluids,17, p. 116101, 2005.

16 of 16

American Institute of Aeronautics and Astronautics 